\title{
Changes in bulk soil affect the disease-suppressive rhizosphere microbiome against Fusarium wilt disease
}

\author{
Lin FU ${ }^{1,2}$, Wu XIONG ${ }^{3}$, Francisco DINI-ANDREOTE, ${ }^{4,5}$, Beibei WANG ${ }^{6}$, Chengyuan TAO ${ }^{1}$, Yunze RUAN \\ Zongzhuan SHEN ${ }^{1}$, Rong LI (凶) ${ }^{1}$, Qirong SHEN ${ }^{1}$ \\ 1 Jiangsu Provincial Key Laboratory of Solid Organic Waste Utilization, Jiangsu Collaborative Innovation Center of Solid Organic Wastes, \\ Education Ministry Engineering Center of Resource-Saving Fertilizers, Nanjing Agricultural University, Nanjing 210095, China \\ 2 School of Life Sciences, Liaoning University, Shenyang 110036, China \\ 3 Ecology and Biodiversity Group, Department of Biology, Institute of Environmental Biology, Utrecht University, Utrecht, 3584 CH, \\ The Netherlands \\ 4 Department of Plant Science, The Pennsylvania State University, University Park, PA 16802, USA \\ 5 Huck Institutes of the Life Sciences, The Pennsylvania State University, University Park, PA 16802, USA \\ 6 Hainan Key Laboratory for Sustainable Utilization of Tropical Bio-Resources, Institute of Tropical Agriculture and Forestry, \\ Hainan University, Haikou 570228, China
}

\begin{abstract}
Harnessing disease-suppressive microbiomes constitutes a promising strategy for optimizing plant growth. However, relatively little information is available about the relationship between bulk and rhizosphere soil microbiomes. Here, the assembly of banana bulk soil and rhizosphere microbiomes was investigated in a monoculture system consisting of bio-organic (BIO) and organic management practices. Applying BIO practice in newly reclaimed fields resulted in a high-efficiency biocontrol rate, thus providing a promising strategy for pre-control of Fusarium wilt disease. The soil microbiota was further characterized by MiSeq sequencing and quantitative PCR. The results indicate that disease suppression was mediated by the structure of a suppressive rhizosphere microbiome with respect to distinct community composition, diversity and abundance. Overall microbiome suppressiveness was primarily related to a particular set of enriched bacterial taxa affiliated with Pseudomonas, Terrimonas, Cupriavidus, Gp6, Ohtaekwangia and Duganella. Finally, structural equation modeling was used to show that the changes in bulk soil bacterial community determined its induced rhizosphere bacterial community, which serves as an important and direct factor in restraining the pathogen. Collectively, this study provides an integrative approach to disentangle the biological basis of disease-suppressive microbiomes in the context of agricultural practice and soil management.
\end{abstract}

Received February 4, 2020; accepted March 13, 2020

Correspondence: lirong@njau.edu.cn
Keywords agricultural practice, bulk soil, disease suppression, rhizosphere ecology

\section{Introduction}

Identifying the important factors that determine ecosystem function is a major challenge, and devising strategies to effectively manipulate them in order to obtain biological benefits is even more demanding ${ }^{[1]}$. The soil microbiome is among the most complex systems for humans to manipulate and is directly connected with plant growth and health ${ }^{[2]}$. Intensive agricultural practices characterized by the use of chemical fertilizers and pesticides are known to alter soil biology by disrupting biotic interactions ${ }^{[3]}$. This can lead to the development of damaging soilborne diseases caused by an unbalanced proliferation of a subset of harmful microbes that encompass a set of plant pathogenic fungi and bacteria ${ }^{[4,5]}$. Among these, Fusarium wilt disease, mainly caused by pathogenic Fusarium oxysporum, is a major factor limiting several cropping systems ${ }^{[6]}$. This pathogen can survive in soil over decades as chlamydospores, and effective methods of control or prevention remain to be developed ${ }^{[7]}$. Thus, the exploration of efficient and practical strategies for $F$. oxysporum control, such as developing strategies that benefit from indigenously developed soil suppressiveness, is urgently needed.

Soil disease suppression is a phenomenon essentially mediated by soil microbiota that has been divided into two categories: general and specific suppression ${ }^{[2]}$. General 
suppression is associated with the biomass, activity and diversity of the soil microbiome ${ }^{[2]}$, and specific suppression is directly associated with a particular microbe (or a consortium of microbes) that directly antagonizes the pathogen in the system ${ }^{[8]}$. Recently, there has been a growing consensus that soil suppressiveness is stimulated by agricultural practices associated with organic matter manipulation ${ }^{[8,9]}$. Hence, a better understanding of abiotic factors structuring disease-suppressive soils is key to developing alternative practices to promote soil health.

The rhizosphere is the zone where a complex microbial network interacts with pathogens and influences the outcome of infection ${ }^{[8]}$. The composition of the resident microbial community in the rhizosphere is of critical importance and has a vital role to the success of pathogen invasion $^{[5,8,10]}$. Previous studies showed that application of a biofertilizer consisting of Bacillus amyloliquefaciens and compost resulted in the control of Fusarium wilt disease by changing the composition and activity of the resident rhizosphere microbes ${ }^{[4,11]}$. It is also important to note that changes imposed in bulk soil, for instance by agricultural practices, have a major role in the composition of rhizosphere communities ${ }^{[12]}$. However, numerous studies have focused on determining suppressive bulk soil microbiomes via integrated soil amendments and there is little information on the relationship between the bulk and rhizosphere microbiomes with respect to disease suppression.

In banana crops worldwide, a particular strain of the fungus Fusarium (F. oxysporum f. sp. cubense) causes Fusarium wilt (or Panama disease) ${ }^{[13]}$. Here, we investigated the biological basis of Fusarium disease suppression induced by organic amendments in banana stands. We hypothesized that the enhanced soil suppressiveness induced by management practices was initially related to bulk soil microbial communities that subsequently determined the suppressive rhizosphere microbiota. A better knowledge of the mechanisms that trigger the immune response of soils can lead to optimized crop management strategies that enhance disease suppression with the goal of improving plant health and securing future crop yields.

\section{Materials and methods}

\subsection{Experimental system}

The experimental site is located in Chengmai County $\left(19^{\circ}\right.$ $65^{\prime} \mathrm{N}, 109^{\circ} 92^{\prime} \mathrm{E}$ ), Hainan Province, one of the major areas of banana production in China. This region has been recently impacted by banana Fusarium wilt. The soil is a Acric Ferralsol with a $\mathrm{pH}$ of 4.83 (1:5 soil:water), organic matter content of $10.2 \mathrm{~g} \cdot \mathrm{kg}^{-1}$, total nitrogen content of $0.79 \mathrm{~g} \cdot \mathrm{kg}^{-1}$, electrical conductivity of $20.9 \mu \mathrm{s} \cdot \mathrm{cm}^{-1}$, available phosphorus of $31.8 \mathrm{mg} \cdot \mathrm{kg}^{-1}$, and available potassium of $64.6 \mathrm{mg} \cdot \mathrm{kg}^{-1}$. The field experiment was conducted over two consecutive years, 2012 and 2013. In February 2012, one area covered with Eucalyptus and Acacia mangium was converted into a banana plantation. Two management regimes in areas of 0.65 ha were established. These regimes were (1) a bio-organic system (BIO), soil amended with bio-organic fertilizer at 12000 and $8000 \mathrm{~kg} \cdot \mathrm{ha}^{-1}$ for the first and second seasons, respectively, lime (Ca-Mg powder) at $3750 \mathrm{~kg} \cdot \mathrm{ha}^{-1}$ each season, and essential mineral fertilizers; and (2) an organic system (CF), soil amended with chicken manure and essential mineral fertilizers. The bacterium Bacillus amyloliquefaciens strain NJN-6 was part of the bio-organic fertilizer treatment and acts as a biocontrol agent against Fusarium wilt ${ }^{[4]}$ at a concentration $1 \times 10^{8} \mathrm{CFU} \cdot \mathrm{g}^{-1}$ dry weight (at the end of the fermentation). In the first year the field was planted with banana tissue culture plantlets (Musa acuminata AAA Cavendish cv. Brazil) at a density of 2400 seedlings ha ${ }^{-1}$. All mother banana plants and unnecessary suckers were cut down two months after harvest, leaving the best sucker per plant for the cropping cycle. Information about the production of the bio-organic fertilizer, other management practices applied in the field and methods of identifying the disease incidence and banana yield has been published previously ${ }^{[7]}$.

\subsection{Sample collection and soil DNA isolation}

Soil samples were collected in September 2013 before harvest. A systematic sampling approach ${ }^{[14]}$ was adopted to collect 10 individual soil samples from each field treatment (Supplementary materials, Fig. S1). Briefly, each field was divided into plots of $10 \mathrm{~m} \times 10 \mathrm{~m}$ in a grid and 10 plots $10 \mathrm{~m}$ apart were selected in a $\mathrm{W}$ shape for sampling. Within each plot, five banana plants at least $4 \mathrm{~m}$ apart were randomly selected and five soil cores $(25 \mathrm{~cm}$ deep $\times$ $2.5 \mathrm{~cm}$ diameter) around a $0.5 \mathrm{~m}$ radius of each banana plant were collected and pooled as composite bulk soil samples. Rhizosphere soils were obtained as previously described $^{[7]}$. The soil samples were immediately stored at $-80^{\circ} \mathrm{C}$ for further DNA isolation. A total of 20 bulk and 20 rhizosphere soil samples were collected (2 fertilizer treatments $\times 10$ replicates per treatment). DNA was isolated using a PowerSoil DNA Isolation Kit (Mobio Laboratories, Carlsbad, CA, USA) following the manufacturer's instructions. The concentration and quality of the extracted DNA were determined using a NanoDrop 2000 spectrophotometer (Thermo Scientific, Waltham, MA, USA).

\subsection{Quantitative PCR analysis}

Copy numbers of marker genes accounting for bacteria ${ }^{[15]}$, fungi ${ }^{[15]}, B$. amyloliquefaciens $\mathrm{NJN}-6^{[4]}$ and $F$. oxysporum $^{[16]}$ were determined using gene-specific primers (Supplementary materials, Table S1) and SYBR Premix Ex 
Taq ${ }^{\mathrm{TM}}$ II (Takara Bio Inc., Kusatsu, Japan) following the manufacturer's instructions. Pseudomonas was detected using a combination of primers and probes (Supplementary materials, Table S1) as described by Bergmark et al. ${ }^{[17]}$. The results are expressed as $\lg$ values (target copy number $\mathrm{g}^{-1}$ soil).

\subsection{MiSeq sequencing and sequence data processing}

The V4 region of the bacterial 16S rRNA gene was amplified from the soil genomic DNA using the primer sets $515 \mathrm{~F}$ and $806 \mathrm{R}^{[18]}$. For fungi the first internal transcribed spacer (ITS) region was amplified by the primer set ITS1F/ ITS2 ${ }^{[19]}$ (Supplementary materials, Table S2). PCR conditions and the protocol for library preparation were conducted as previously described ${ }^{[11]}$. The libraries were sequenced on an Illumina MiSeq platform at Novogene Bioinformatics Technology Co., Ltd. (Beijing, China). The resulting raw sequences were processed using QIIME (1.8.0) to demultiplex and remove adaptor and primer sequences $^{[20]}$. Sequences were then paired using FLASH v1.2.7. Merged sequences were filtered and clustered using USEARCH, UPARSE, and Perl scripts to remove lowquality sequences ${ }^{[21]}$. OTU tables were generated using 97\% nucleotide identity and sequences were annotated using the RDP naïve Bayesian classifier for bacteria ${ }^{[22]}$ and UNITE for fungal sequences ${ }^{[23]}$. All sequence data are available through accession number SRP072164 in the NCBI Sequence Read Archive.

\subsection{Statistical analysis}

All statistical tests were conducted in $\mathrm{R} v 3.2 .0^{[24]}$ and considered significant at $P<0.05$. Non-normal data were square-root or log-transformed to meet test assumptions. Significant differences between samples were determined by two-tailed Student's or Welch's $t$-tests or the Wilcoxon rank sum test. Prior to statistical tests and community metrics inferences the original OTU tables were rarefied at depths of 8397 sequences for bacteria and 8955 sequences for fungi per sample.

\subsection{1 $\alpha$ - and $\beta$-diversity metrics}

$\alpha$-diversity (the number and abundance of taxa within communities) and $\beta$-diversity (compositional dissimilarity between communities) metrics were adopted to characterize the soil microbiomes. The number of observed OTUs $\left(\mathrm{S}_{\text {obs }}\right)$ and Faith's phylogenetic diversity (PD) index were determined using Mothur ${ }^{[25]}$. $\beta$-diversity of both bacterial and fungal communities was determined using non-metric multidimensional scaling (NMDS) analysis based on the Bray-Curtis distance matrices in Mothur ${ }^{[25]}$. Analysis of similarity (ANOSIM) $)^{[26]}$ and permutational multivariate analysis of variance (PERMANOVA, 999 permutations) were conducted to test for significant differences among microbial composition of samples using the "vegan" package in R. Multiple regression tree (MRT) analysis, based on the Bray-Curtis distance metric, was conducted to evaluate the relative influences of management and the soil compartment on the microbiome using "vegan", "mvpart", and "MVPARTwrap" packages in R. Differences in the relative abundance of bacterial and fungal genera between $\mathrm{BIO}$ and $\mathrm{CF}$ were conducted using STAMP ${ }^{[27]}$. $P$-values were calculated using a two-sided Welch's $t$-test and confidence intervals were calculated using Welch's inverted method. The false discovery rate (FDR) was calculated using Benjamini-Hochberg with $q<0.05$. Spearman correlations were used to relate the relative abundance of specific bacterial taxa with copy numbers of $F$. oxysporum using the package "stat" in R.

\subsubsection{Structural equation modeling}

Structural equation modeling (SEM) was constructed to tease apart indirect and direct effects of bulk soil microbiota on rhizosphere microbiota in a multivariate approach using the packages "sem" and "lavaan" in R. The first axis of NMDS analysis comprising Pseudomonas, Duganella, Gp6, Terrimonas, Cupriavidus, and Ohtaekwangia was used to quantify rhizosphere-responsive OTUs. We quantified $F$. oxysporum and NJN-6 abundances using targeted gene copy numbers. The bacterial and fungal community composition was quantified using the third and second axes of the NMDS, respectively. All variables were standardized using the scale function in $\mathrm{R}$. Goodness-of-fit was evaluated on the basis of a $\chi^{2}$ test $(P>0.05$ indicates a good model fit).

\section{Results}

3.1 Effects of soil management on Fusarium wilt disease incidence and fruit yield

We observed an overall increase in Fusarium wilt disease incidence in the second year in both BIO and CF systems. However, the rate of disease increase differed between systems. The rate of disease in the BIO treatment increased from $2.9 \%$ to $13.2 \%$ while the rate in $\mathrm{CF}$ increased from $10.1 \%$ to $50.3 \%$ (Fig. 1(a)). These results indicate that the BIO system greatly contributed to a lower disease incidence in the newly converted banana fields. In addition, the BIO system significantly enhanced the average banana yield per plant compared to the yield from the CF system (the BIO system yield was $16.0 \%$ higher than that in the $\mathrm{CF}$ system in the first year and $18.9 \%$ higher in the second year) (Fig. 1(b)). 

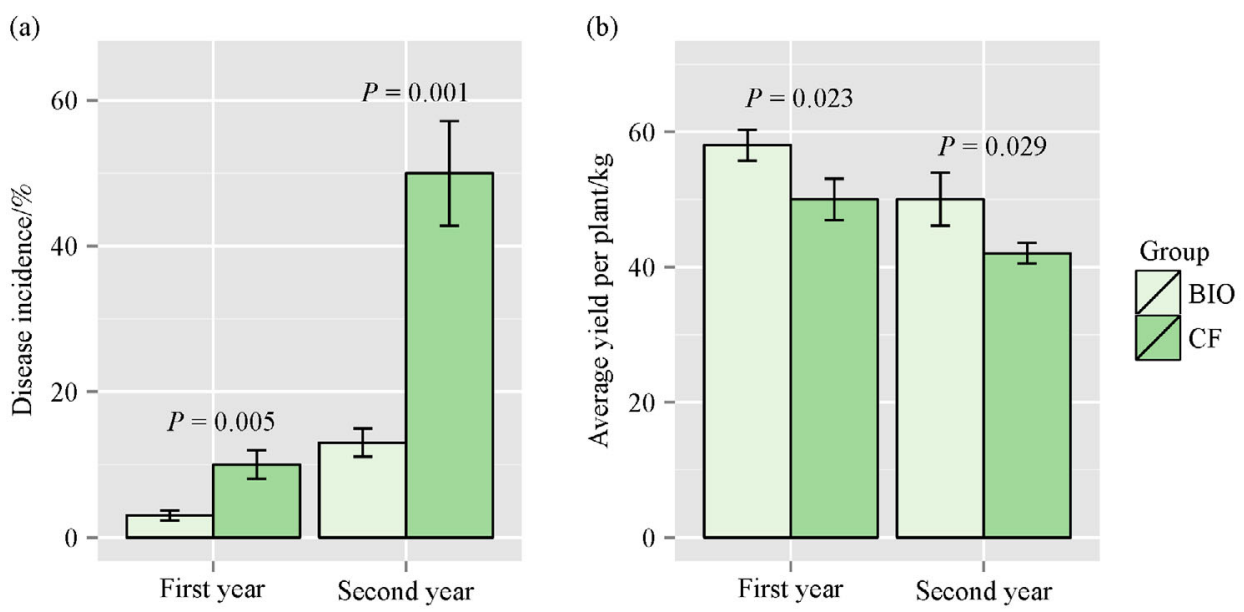

Fig. 1 Banana Fusarium wilt disease incidence (a) and yield in BIO and CF systems (b). $P$-values were determined by two-tailed Student's $t$-test. Error bars are standard errors, $n=3$.

\subsection{Differences in community abundance and $\alpha$-diversity}

qPCR results showed that significantly more bacteria (Welch's $t$-test, $P<0.001$ ) and fewer fungi were detected in BIO bulk soil (BIOB) than in the bulk soil from $\mathrm{CF}$ (CFB), and results from rhizosphere soil samples showed a similar tendency (Fig. 2). The BIO system led to significantly higher bacterial diversity (Faith's PD) (Student's $t$-test, $P<0.05$ ) in BIOB compared to that found in $\mathrm{CFB}$, however there were no significant trends observed for bacteria in rhizosphere soil and fungi in bulk and rhizosphere soils (Fig. 2). Furthermore, a significantly higher bacterial richness $\left(\mathrm{S}_{\mathrm{obs}}\right)$ was observed in soils collected from BIO compared to CF (Welch's $t$-test, $P<0.01)$. Fungal results showed the opposite trends, with rhizosphere soils showing a significant difference (Fig. 2).

\subsection{Changes in microbiome $\beta$-diversity}

As shown in Fig. 3(a) and 3(b), NMDS plots showed differences in the bacterial and fungal community composition in BIO and CF (PERMANOVA, $P<0.001$; ANOSIM, $P<0.001$; Supplementary materials, Table S3). BIO was distinctly separated from CF along the NMDS1NMDS2 plane and NMDS1-NMDS3 plane, respectively, for bacteria and fungi. The fertilization scheme and soil compartment explained $28 \%$ and $14 \%$ of the variation, respectively, for bacteria and $14 \%$ and $16 \%$ for fungi (Supplementary materials, Table S3). MRT analysis showed similar trends in which the fertilization scheme accounted for the largest proportion of variation explaining the microbiome composition, followed by the soil compartment (Fig. 3(c) and 3(d)).
3.4 Depicting microbial taxa potentially associated with disease suppression

After STAMP analysis, significant differences in bulk and rhizosphere soil microbial composition between BIO and CF were observed (Supplementary materials, Fig. S2 and Fig. S3). Significant increases were observed in OTUs associated with Cupriavidus, Duganella, Terrimonas, Gp6, Gp4, Nitrospira, Ohtaekwangia, Pseudomonas, Gemmatimonas, Flavobacterium, and Planctomyces (BenjaminiHochberg FDR, $q<0.05)$ in rhizosphere samples in the BIO system. Of these, Terrimonas, Pseudomonas, Cupriavidus, Gp6, Ohtaekwangia, and Duganella were also enriched in the bulk soil (Benjamini-Hochberg FDR, $q<0.05$ ) (Supplementary materials, Fig. S2). Regarding the fungi, only one unidentified genus belonging to Sordariomycetes differed in relative abundance between BIOB and CFB (Supplementary materials, Fig. S3). However, in the rhizosphere, significantly higher relative abundances of two taxa, Sordariomycetes (unidentified) and Microascaceae (unidentified), and significantly lower relative abundances of Aspergillus, Sordariaceae (unidentified), Fusarium, Penicillium, Retroconis, Purpureocillium, Trichocomaceae (unidentified), Humicola, Clonostachys, Chaetomiaceae (unidentified), Chaetomium, Acaulospora, Mortierella, Calonectria, Trichocladium, Scedosporium and Sordariales (unidentified) (Benjamini-Hochberg FDR, $q<0.05$ ), were found in BIO than in CF. Moreover, significantly lower abundance of $F$. oxysporum (Student's $t$ test, $P<0.001)$ and higher abundance of Pseudomonas (Welch's $t$-test, $P<0.001$ ) in BIO rhizosphere samples were validated using qPCR (Supplementary materials, Fig. S4). Although a higher relative abundance of Bacillus was not observed, the biocontrol agent $B$. amyloliquefaciens 

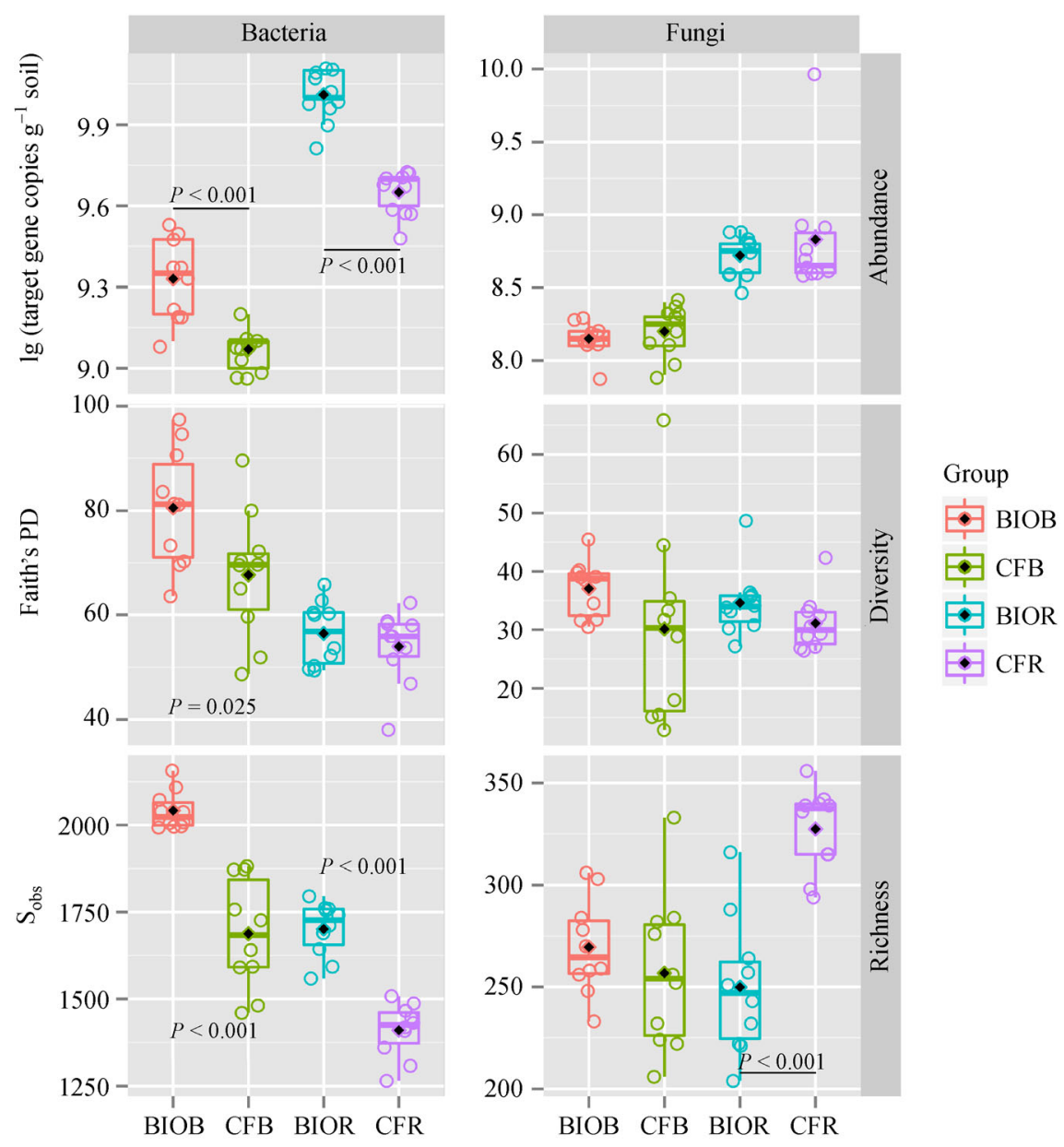

Fig. 2 Bacterial and fungal abundance (target copy number), richness $\left(\mathrm{S}_{\mathrm{obs}}\right)$ and diversity (Faith's PD) index. Box plots show the median (thick colored line), mean (blacked diamond), the first quartile (lower box bound), the third quartile (upper box bound), the range of data values that deviate from the box no more than 1.5 times the height of the box (vertical lines). BIOB, bulk soil from bio-organic system; CFB, bulk soil from organic system; BIOR, rhizosphere soil from bio-organic system; CFR, rhizosphere soil from organic system. $P$-values over paired columns indicate that the means are significantly different according to the two-tailed Student's or Welch's $t$-test or the Wilcoxon rank sum test $(P<0.05)$.

NJN-6 was successfully detected in both bulk and rhizosphere soil samples (Supplementary materials, Fig. S4).

Spearman correlations were conducted between $F$. oxysporum copy number and the relative abundances of specific bacterial genera that significantly responded to BIO management. These were potentially associated with wilt disease suppression (Fig. 4). As shown in Fig. 4, the $F$. oxysporum copy number was negatively correlated with Pseudomonas $(r=-0.81, P<0.0001)$, Duganella $(r=$ $-0.75, P=0.0003), G p 6(r=-0.65, P=0.03)$, Terrimonas $(r=-0.74, P=0.0003)$, Cupriavidus $(r=-0.81$, $P<0.0001)$ and Ohtaekwangia $(r=-0.57, P=0.01)$.

\section{Discussion}

A practical fertilization strategy, namely application of $\mathrm{BIO}$ in the newly reclaimed fields, gave highly efficient biocontrol efficacy in the pre-control of Fusarium wilt disease. Our results are consistent with previous studies in which the continuous application of BIO to recently established agricultural sites or continuous plantation with banana both produced enhanced suppression of Fusarium wilt disease ${ }^{4,10]}$. The field results provide a unique opportunity to explore in more detail the relationships between the disease-suppressive bulk soil and rhizosphere microbiomes.

Higher bacterial abundance (qPCR), diversity (Faith's $\mathrm{PD})$, and richness $\left(\mathrm{S}_{\mathrm{obs}}\right)$ and lower fungal abundance, diversity, and richness were observed in biofertilizeramended soil, and results from rhizosphere soil samples showed trends similar to those in bulk soil. These results are in line with a previous study in which, compared to the control, the $\alpha$-diversity of bacteria significantly increased whereas fungal diversity decreased after two years of biofertilizer application ${ }^{[10]}$. This illustrates the potential 

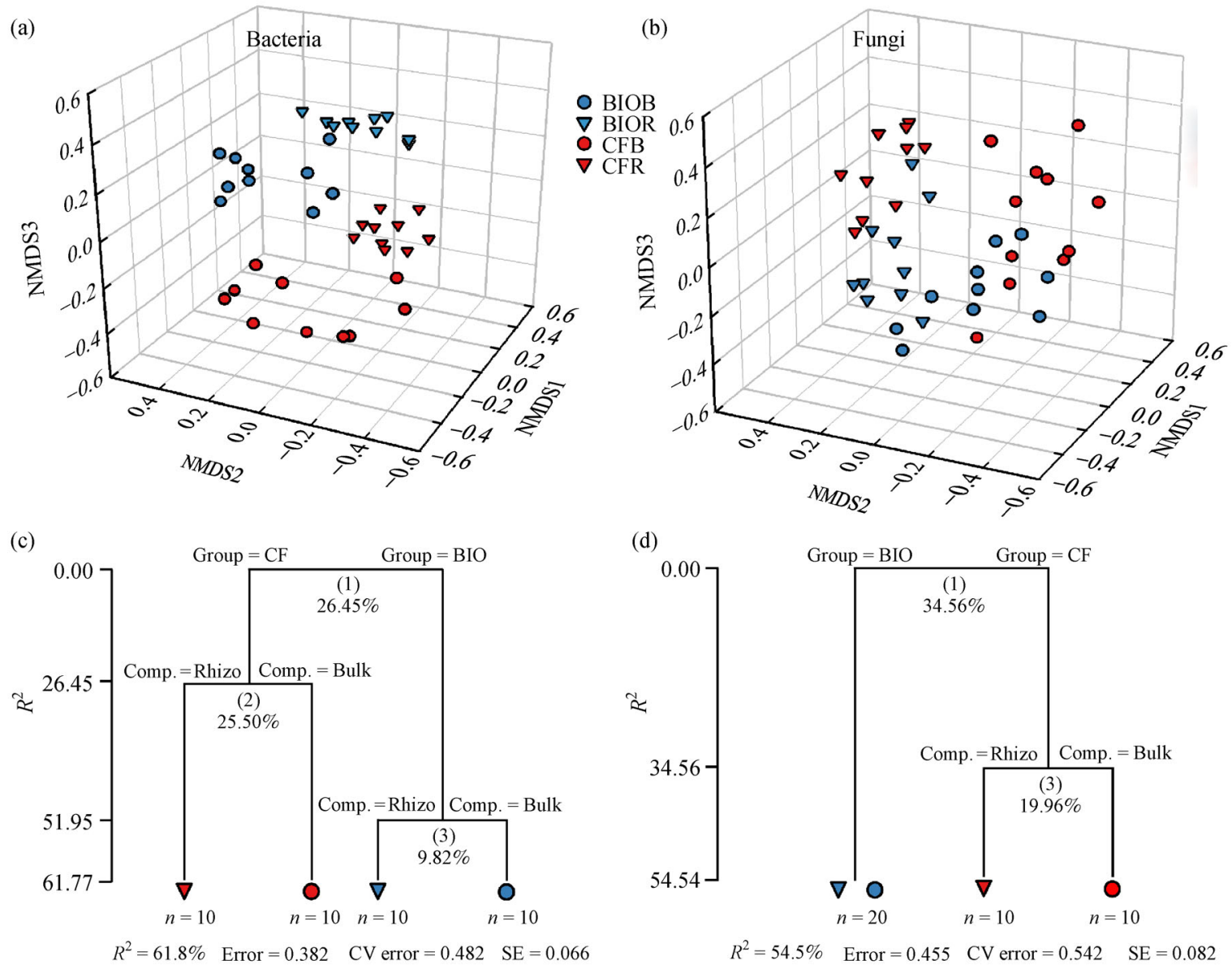

Fig. 3 (a, b) Non-metric multidimensional scaling (NMDS) ordinations and (c, d) multivariate regression tree (MRT) analysis. BIOB, bulk soil from bio-organic system; CFB, bulk soil from organic system; BIOR, rhizosphere soil from bio-organic system; CFR, rhizosphere soil from organic system. The identity and number of soil samples included in the analysis are shown under the tree by symbols with different shapes and colors. Numbers under the crosses of each split indicate percentages of variance explained by the split. The $R^{2}$, error, cross-validation error (CV error), and standard error (SE) of MRT analysis are listed under the trees.

boost of soil bacterial activity by novel fertilization strategies which was ultimately reflected in its variation. Higher bacterial diversity has been observed to be advantageous in soil disease suppression ${ }^{[28]}$, and microbial diversity is becoming progressively recognized as a property that hinders pathogen establishment in soils ${ }^{[29]}$. Thus, our findings indicate that higher bacterial community abundance, diversity and richness in the rhizosphere may have created a more phylogenetically diverse community that occupies the majority of the available niches in soil, thus restricting the further multiplication and development of the pathogen in the system.

NMDS and MRT both revealed significant differences in bulk soil bacterial and fungal community composition between the different fertilization strategies. This finding indicates that different soil amendments induce differences in microbial composition ${ }^{[30]}$, and PGPR-containing bio-organic fertilizers have been broadly confirmed to have a significant effect in shaping soil microbial communities $^{[5,10]}$. Importantly, we found similar variation in bacterial and fungal communities in rhizosphere and bulk soil samples. As previously shown ${ }^{[31,32]}$, we also found that the rhizosphere microbial composition was largely dependent on the composition of the bulk soil microbiota. In addition, fertilization and the soil compartment had important roles in determining the composition of the microbiome. Consistent with our results, fertilization has been reported to influence microbial composition (structure) in the soil and rhizosphere in different crop systems $^{[4,33,34]}$. Hence, we show that the fertilization pattern initially altered the community composition of the bulk soil microbiome and this further resulted in changes to their respective rhizosphere microbiomes.

The abundance of the pathogen was negatively correlated with bacteria belonging to the genera Pseudomonas, Duganella, Cupriavidus, Terrimonas, Ohtaekwangia, and 

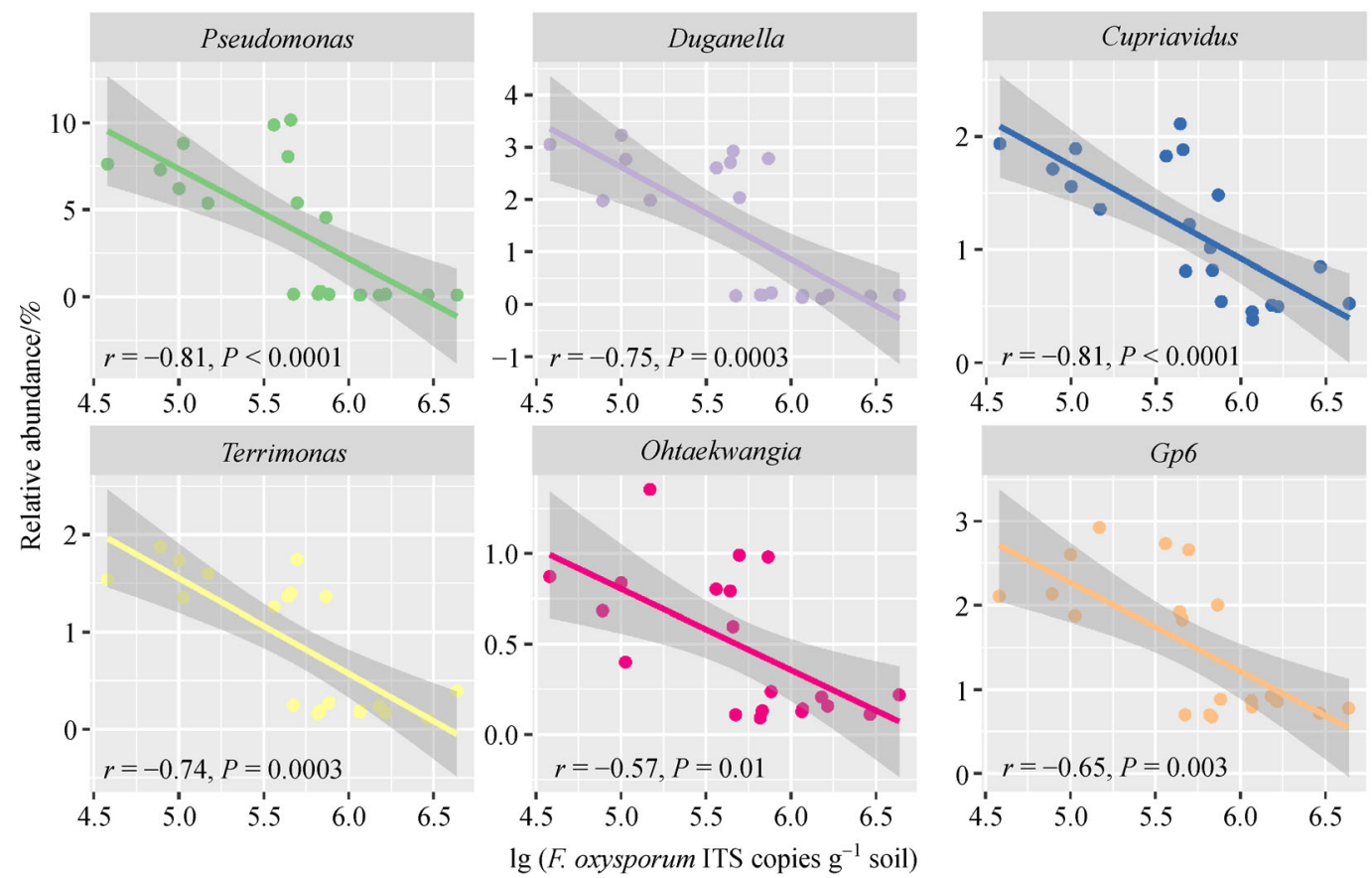

Fig. 4 Spearman rank correlations between copy numbers of Fusarium oxysporum and specific bacterial relative abundances in the BIO system.

Gp6, all of which were enriched in both bulk soil and rhizosphere samples in BIO. Pseudomonas has been described as having a wide spectrum of biological activities $^{[35]}$ such as antibiosis, induced systemic resistance and plant growth promotion, and this genus has already been noted to be associated with the suppression of Fusarium wilt on banana ${ }^{[36]}$. Duganella, a member of the family Oxalobacteraceae, is well-known for its antifungal effects including against Fusarium, and the antifungal activity is most likely induced by the synthesis of chitinase ${ }^{[37]}$. Although no antagonistic activity against F. oxysporum was observed, interplay of Cupriavidus with some other non-antagonistic bacteria can significantly contribute to the development of suppression against $F$. oxysporum both in vitro and in plant ${ }^{[38]}$. Furthermore, Cupriavidus can produce lipopeptide siderophores such as taiwachelin ${ }^{[39]}$ which may mediate in competition for iron and induced resistance to suppress pathogenic fungi ${ }^{[40]}$. Although there has been no report that the genera Terrimonas and Ohtaekwangia possess specific biocontrol activity against $F$. oxysporum, these two genera were identified as key groups that confer soil disease-suppressiveness against Fusarium wilt pathogen invasion ${ }^{[41]}$. In addition, species in the Chitinophagaceae and Cytophagales were reported to be producers of chitinolytic enzymes which are involved in fungal cell wall lysis and chitin degradation ${ }^{[42]}$. The Gp6 group was reported to occur in higher relative abundance in potato common scab and banana Fusarium wilt disease-suppressive soils than in disease-conducive soils ${ }^{[4,41,43]}$. Despite this indication, the actual role of Gp6 influencing disease suppression is still unknown. Only one unidentified genus belonging to the Sordariomycetes differed in relative abundance between $\mathrm{BIO}$ and CF. However, in the rhizosphere, a significantly lower relative abundance of Fusarium was observed in $\mathrm{BIO}$ compared to CF. Moreover, the abundance of Pseudomonas was further detected by qPCR and demonstrated a similar trend as that observed in the sequencing results. Hence, in addition to the microbes amended with the biofertilizers ( $B$. amyloliquefaciens), which can be detected both in BIO bulk and rhizosphere soils, the increased relative abundances of these indigenous (and potentially antagonistic) species may likely be involved in Fusarium wilt disease suppression, for instance through taxa-specific mechanisms.

\section{Conclusions}

Here, we have demonstrated efficient Fusarium wilt disease suppression capacity in newly converted fields with the application of bio-organic fertilizer. As a summary of the variation in the SEM model (Fig. 5), the changes in bulk soil bacterial community determined its induced rhizosphere bacterial community, and serves as an important and direct factor in restraining the pathogen. In addition, biocontrol agents and responsive bacterial genera both act as direct disease suppression indicators. 


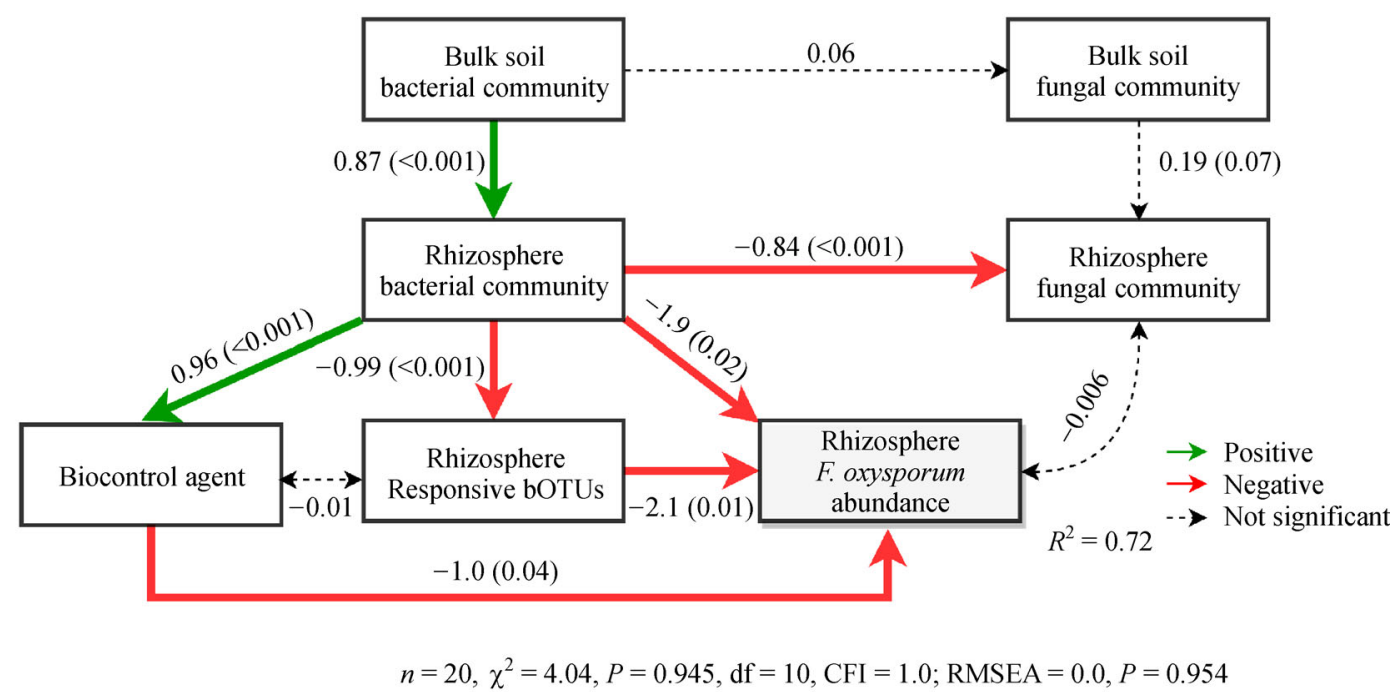

Fig. 5 Structural equation modeling integrating direct and indirect relationships of bulk soil and rhizosphere microbiomes (bacteria and fungi), differentially occurring bacterial OTUs (bOTUs), the abundance of the biocontrol agent NJN-6, and the abundance of Fusarium oxysporum. Arrows indicate the flow of causality. Solid arrows represent statistically significant relationships $(P<0.05)$, and dashed black arrows are nonsignificant relationships. Numbers adjacent to arrows are standardized path coefficients and bootstrap $P$-values. The arrow width is proportional to the strength of path coefficients. $R^{2}$ denotes the proportion of variance explained. Goodness-of-fit statistics for the model are shown at the bottom

Collectively, understanding how different organic amendments affect the assembly and functioning of microbiomes is the basis for exploring robust soil practices to manipulate bulk and rhizosphere soil systems. We also propose that the beneficial effects of taxa-specific suppression should be considered in the design of bio-organic fertilizers and their use in sustainable strategies for plant protection against diverse disease agents.

Supplementary materials The online version of this article at https://doi. org/10.15302/J-FASE-2020328 contains supplementary materials (Figs. S1-S4; Tables S1-S3).

Acknowledgements This research was funded by the National Key Research and Development Program (2017YFD0202101), the National Natural Science Foundation of China (31672239 and 31972509), the 111 project (B12009), the Priority Academic Program Development of the Jiangsu Higher Education Institutions (PAPD), and the Innovative Research Team Development Plan of the Ministry of Education of China (IRT_17R56).

Compliance with ethics guidelines Lin Fu, Wu Xiong, Francisco DiniAndreote, Beibei Wang, Chengyuan Tao, Yunze Ruan, Zongzhuan Shen, Rong $\mathrm{Li}$, and Qirong Shen declare that they have no conflicts of interest or financial conflicts to disclose.

This article does not contain any studies with human or animal subjects performed by any of the authors.

\section{References}

1. Toju H, Peay K G, Yamamichi M, Narisawa K, Hiruma K, Naito K,
Fukuda S, Ushio M, Nakaoka S, Onoda Y, Yoshida K, Schlaeppi K, Bai Y, Sugiura R, Ichihashi Y, Minamisawa K, Kiers E T. Core microbiomes for sustainable agroecosystems. Nature Plants, 2018, 4(5): $247-257$

2. Raaijmakers J M, Mazzola M. Soil immune responses. Science, 2016, 352(6292): 1392-1393

3. Matson P A, Parton W J, Power A G, Swift M J. Agricultural intensification and ecosystem properties. Science, 1997, 277(5325): 504-509

4. Fu L, Penton C R, Ruan Y Z, Shen Z Z, Xue C, Li R, Shen Q R. Inducing the rhizosphere microbiome by biofertilizer application to suppress banana Fusarium wilt disease. Soil Biology \& Biochemistry, 2017, 104: 39-48

5. Liu H J, Xiong W, Zhang R F, Hang X N, Wang D S, Li R, Shen Q R. Continuous application of different organic additives can suppress tomato disease by inducing the healthy rhizospheric microbiota through alterations to the bulk soil microflora. Plant and Soil, 2018, 423(1-2): 229-240

6. Klein E, Katan J, Gamliel A. Soil suppressiveness by organic amendment to Fusarium disease in cucumber: effect on pathogen and host. Phytoparasitica, 2016, 44(2): 239-249

7. Fu L, Ruan Y, Tao C, Li R, Shen Q. Continous application of bioorganic fertilizer induced resilient culturable bacteria community associated with banana Fusarium wilt suppression. Scientific Reports, 2016, 6(1): 27731

8. Raaijmakers J M, Paulitz T C, Steinberg C, Alabouvette C, MoënneLoccoz Y. The rhizosphere: a playground and battlefield for soilborne pathogens and beneficial microorganisms. Plant and Soil, 
2009, 321(1-2): 341-361

9. Bonanomi G, Antignani V, Capodilupo M, Scala F. Identifying the characteristics of organic soil amendments that suppress soilborne plant diseases. Soil Biology \& Biochemistry, 2010, 42(2): 136-144

10. Shen Z Z, Ruan Y Z, Xue C, Zhang J, Li R, Shen Q R. Rhizosphere microbial community manipulated by 2 years of consecutive biofertilizer application associated with banana Fusarium wilt disease suppression. Biology and Fertility of Soils, 2015, 51(5): $553-562$

11. Shen Z Z, Ruan Y Z, Wang B B, Zhong S T, Su L X, Li R, Shen Q R. Effect of biofertilizer for suppressing Fusarium wilt disease of banana as well as enhancing microbial and chemical properties of soil under greenhouse trial. Applied Soil Ecology, 2015, 93: 111-119

12. Mendes L W, Kuramae E E, Navarrete A A, van Veen J A, Tsai S M. Taxonomical and functional microbial community selection in soybean rhizosphere. ISME Journal, 2014, 8(8): 1577-1587

13. Butler D. Fungus threatens top banana. Nature, 2013, 504(7479): 195-196

14. Wang J F, Stein A, Gao B B, Ge Y. A review of spatial sampling. Spatial Statistics, 2012, 2(1): 1-14

15. Fierer N, Jackson J A, Vilgalys R, Jackson R B. Assessment of soil microbial community structure by use of taxon-specific quantitative PCR assays. Applied and Environmental Microbiology, 2005, 71(7): $4117-4120$

16. Jiménez-Fernández $\mathrm{D}$, Montes-Borrego $\mathrm{M}$, Navas-Cortés $\mathrm{J}$ A, Jiménez-Díaz R M, Landa B B. Identification and quantification of Fusarium oxysporum in planta and soil by means of an improved specific and quantitative PCR assay. Applied Soil Ecology, 2010, 46 (3): 372-382

17. Bergmark L, Poulsen P H B, Al-Soud W A, Norman A, Hansen L H, Sørensen S J. Assessment of the specificity of Burkholderia and Pseudomonas qPCR assays for detection of these genera in soil using 454 pyrosequencing. FEMS Microbiology Letters, 2012, 333 (1): $77-84$

18. Caporaso J G, Lauber C L, Walters W A, Berg-Lyons D, Lozupone C A, Turnbaugh P J, Fierer N, Knight R. Global patterns of $16 \mathrm{~S}$ rRNA diversity at a depth of millions of sequences per sample. Proceedings of the National Academy of Sciences of the United States of America, 2011, 108(Suppl 1): 4516-4522

19. McGuire K L, Payne S G, Palmer M I, Gillikin C M, Keefe D, Kim S J, Gedallovich S M, Discenza J, Rangamannar R, Koshner J A, Massmann A L, Orazi G, Essene A, Leff J W, Fierer N. Digging the New York City Skyline: soil fungal communities in green roofs and city parks. PLoS One, 2013, 8(3): e58020

20. Caporaso J G, Kuczynski J, Stombaugh J, Bittinger K, Bushman F D, Costello E K, Fierer N, Peña A G, Goodrich J K, Gordon J I, Huttley G A, Kelley S T, Knights D, Koenig J E, Ley R E, Lozupone C A, McDonald D, Muegge B D, Pirrung M, Reeder J, Sevinsky J R, Turnbaugh P J, Walters W A, Widmann J, Yatsunenko T, Zaneveld J, Knight R. QIIME allows analysis of high-throughput community sequencing data. Nature Methods, 2010, 7(5): 335-336

21. Edgar R C. UPARSE: highly accurate OTU sequences from microbial amplicon reads. Nature Methods, 2013, 10(10): 996-998

22. Wang Q, Garrity G M, Tiedje J M, Cole J R. Naïve Bayesian classifier for rapid assignment of rRNA sequences into the new bacterial taxonomy. Applied and Environmental Microbiology,
2007, 73(16): 5261-5267

23. Kõljalg U, Nilsson R H, Abarenkov K, Tedersoo L, Taylor A F S, Bahram M, Bates S T, Bruns T D, Bengtsson-Palme J, Callaghan T M, Douglas B, Drenkhan T, Eberhardt U, Dueñas M, Grebenc T, Griffith G W, Hartmann M, Kirk P M, Kohout P, Larsson E, Lindahl B D, Lücking R, Martín M P, Matheny P B, Nguyen N H, Niskanen T, Oja J, Peay K G, Peintner U, Peterson M, Põldmaa K, Saag L, Saar I, Schüßler A, Scott J A, Senés C, Smith M E, Suija A, Taylor D L, Telleria M T, Weiss M, Larsson K H. Towards a unified paradigm for sequence-based identification of fungi. Molecular Ecology, 2013, 22(21): 5271-5277

24. The R Core Team. R: A language and environment for statistical computing. Vienna, Austria: R Foundation for Statistical Computing, 2018

25. Schloss P D, Westcott S L, Ryabin T, Hall J R, Hartmann M, Hollister E B, Lesniewski R A, Oakley B B, Parks D H, Robinson C J, Sahl J W, Stres B, Thallinger G G, Van Horn D J, Weber C F. Introducing mothur: open-source, platform-independent, community-supported software for describing and comparing microbial communities. Applied and Environmental Microbiology, 2009, 75 (23): 7537-7541

26. Clarke K R. Non-parametric multivariate analyses of changes in community structure. Australian Journal of Ecology, 1993, 18(1): 117-143

27. Parks D H, Tyson G W, Hugenholtz P, Beiko R G. STAMP: statistical analysis of taxonomic and functional profiles. Bioinformatics, 2014, 30(21): 3123-3124

28. Garbeva P, Postma J, van Veen J A, van Elsas J D. Effect of aboveground plant species on soil microbial community structure and its impact on suppression of Rhizoctonia solani AG3. Environmental Microbiology, 2006, 8(2): 233-246

29. van Elsas J D, Chiurazzi M, Mallon C A, Elhottovā D, Krištůfek V, Salles J F. Microbial diversity determines the invasion of soil by a bacterial pathogen. Proceedings of the National Academy of Sciences of the United States of America, 2012, 109(4): 1159-1164

30. Whitman T, Pepe-Ranney C, Enders A, Koechli C, Campbell A, Buckley D H, Lehmann J. Dynamics of microbial community composition and soil organic carbon mineralization in soil following addition of pyrogenic and fresh organic matter. ISME Journal, 2016, 10(12): 2918-2930

31. Ridder-Duine A S, Kowalchuk G A, Gunnewiek P J A K, Smant W, van Veen J A, Boer W. Rhizosphere bacterial community composition in natural stands of Carex arenaria (sand sedge) is determined by bulk soil community composition. Soil Biology \& Biochemistry, 2005, 37(2): 349-357

32. Bakker M G, Chaparro J M, Manter D K, Vivanco J M. Impacts of bulk soil microbial community structure on rhizosphere microbiomes of Zea mays. Plant and Soil, 2015, 392(1-2): 115-126

33. Hartmann M, Frey B, Mayer J, Mäder P, Widmer F. Distinct soil microbial diversity under long-term organic and conventional farming. ISME Journal, 2015, 9(5): 1177-1194

34. Xiong W, Guo S, Jousset A, Zhao Q Y, Wu H S, Li R, Kowalchuk G A, Shen Q R. Bio-fertilizer application induces soil suppressiveness against Fusarium wilt disease by reshaping the soil microbiome. Soil Biology \& Biochemistry, 2017, 114: 238-247

35. Raaijmakers J M, Weller D M. Exploiting genotypic diversity of 
2,4-diacetylphloroglucinol-producing Pseudomonas spp.: characterization of superior root-colonizing P. fluorescens strain Q8r1-96. Applied and Environmental Microbiology, 2001, 67(6): 2545-2554

36. Fishal E M M, Meon S, Yun W M. Induction of tolerance to Fusarium Wilt and defense-related mechanisms in the plantlets of susceptible berangan banana pre-inoculated with Pseudomonas sp. (UPMP3) and Burkholderia sp. (UPMB3). Agricultural Sciences in China, 2010, 9(8): 1140-1149

37. Haack F S, Poehlein A, Kröger C, Voigt C A, Piepenbring M, Bode H B, Daniel R, Schäfer W, Streit W R. Molecular keys to the Janthinobacterium and Duganella spp. interaction with the plant pathogen Fusarium graminearum. Frontiers in Microbiology, 2016, 7: 1668

38. Fujiwara K, Iida Y, Someya N, Takano M, Ohnishi J, Terami F, Shinohara M. Emergence of antagonism against the pathogenic fungus Fusarium oxysporum by interplay among non-antagonistic bacteria in a hydroponics using multiple parallel mineralization.
Journal of Phytopathology, 2016, 164(11-12): 853-862

39. Kreutzer M F, Nett M. Genomics-driven discovery of taiwachelin, a lipopeptide siderophore from Cupriavidus taiwanensis. Organic \& Biomolecular Chemistry, 2012, 10(47): 9338-9343

40. Neilands J B, Leong S A. Siderophores in relation to plant growth and disease. Annual Review of Plant Physiology, 1986, 37(1): 187208

41. Ou Y, Penton C R, Geisen S, Shen Z, Sun Y, Lv N, Wang B, Ruan Y, Xiong W, Li R, Shen Q. Deciphering underlying drivers of disease suppressiveness against pathogenic Fusarium oxysporum. Frontiers in Microbiology, 2019, 10: 2535

42. Reichenbach H. The order cytophagales. In: Balows A, Trüper H G, Dworkin M, Harder W, Schleifer K, eds. The prokaryotes. New York: Springer, 2006, 549-590

43. Rosenzweig N, Tiedje J M, Quensen J F 3rd, Meng Q, Hao J J. Microbial communities associated with potato common scabsuppressive. Plant Disease, 2012, 96(5): 718-725 